\title{
Ileal Mucosal Diaphragm Causing Small Bowel Obstruction
}

\author{
Farooq P. Agha ${ }^{1}$ and Jesse J. Jenkins ${ }^{2}$ \\ 1 Department of Radiology, University of Michigan Hospital, Ann Arbor, Michigan; and \\ 2 Department of Pathology, West Virginia University Hospital, Morgantown, West Virginia, USA
}

\begin{abstract}
A case of small bowel obstruction in an adult due to ileal mucosal diaphragm is reported. This unusual cause of small bowel obstruction to the best of our knowledge has not been previously reported. The cause of intestinal diaphragm remains uncertain.
\end{abstract}

Key words: Intestine, obstruction - Ileum, mucosal diaphragm.

The occurrence of mucosal diaphragm in the esophagus, stomach, and duodenum has been well documented in the literature [1-6]. The following case illustrates that a similar lesion may also occur in the ileum and lead to obstruction of the small bowel.

\section{Case Report}

A 18-year-old man presented with acute abdominal pain and vomiting of 4 hours' duration. There was no past history of significant disease, trauma, or surgery. Family history was unremarkable.

Physical examination revealed an otherwise healthy man in acute abdominal distress. The abdomen was slightly distended with no localizing tenderness. The bowel sounds were hyperactive. Review of other systems was normal. Laboratory data and urinanalysis were unremarkable. An abdominal radiograph revealed distended loops of small bowel with multiple air-fluid levels (Fig. 1) consistent with mechanical small bowel obstruction. At laparotomy a segment of small bowel 8 feet proximal to the ileocecal valve revealed intrinsic obstruction. A $12 \mathrm{~cm}$ long segment of ileum was resected, and end-to-end anastomosis and appendectomy were performed. The gross specimen (Fig. 2A) consisted of a $12 \mathrm{~cm}$ long segment of ileum varying from 3 to $7 \mathrm{~cm}$ in internal circumference. The serosal surface was smooth and glistening. A soft $5 \times 6 \times 6 \mathrm{~cm}$

Address reprint requests to: Farooq P. Agha, M.D., Department of Radiology, University of Michigan Hospital, Ann Arbor, MI 48109, USA bulge was palpable in the central part of the specimen. When the specimen was opened longitudinally, the soft central mass was seen to be composed of 3 almost circumferential $0.5-1.5 \mathrm{~cm}$ high mucosal folds forming a partially obstructing diaphragm or valve-like structure. The mucosal surface appeared normal. Histopathologic study (Fig. 2B) revealed 3 high valve-like structures composed of large folds of mucosa and submucosa with abundant smooth muscle bundles which were focally continuous with the inner circular muscle layer of the muscularis externa. The mucosa of these high folds was normal. The pathology report was 3 valve-like high circumferential mucosal folds consistent with ileal diaphragm. The appendix revealed focal luminal fibrous obliteration with no evidence of acute inflammatory process.

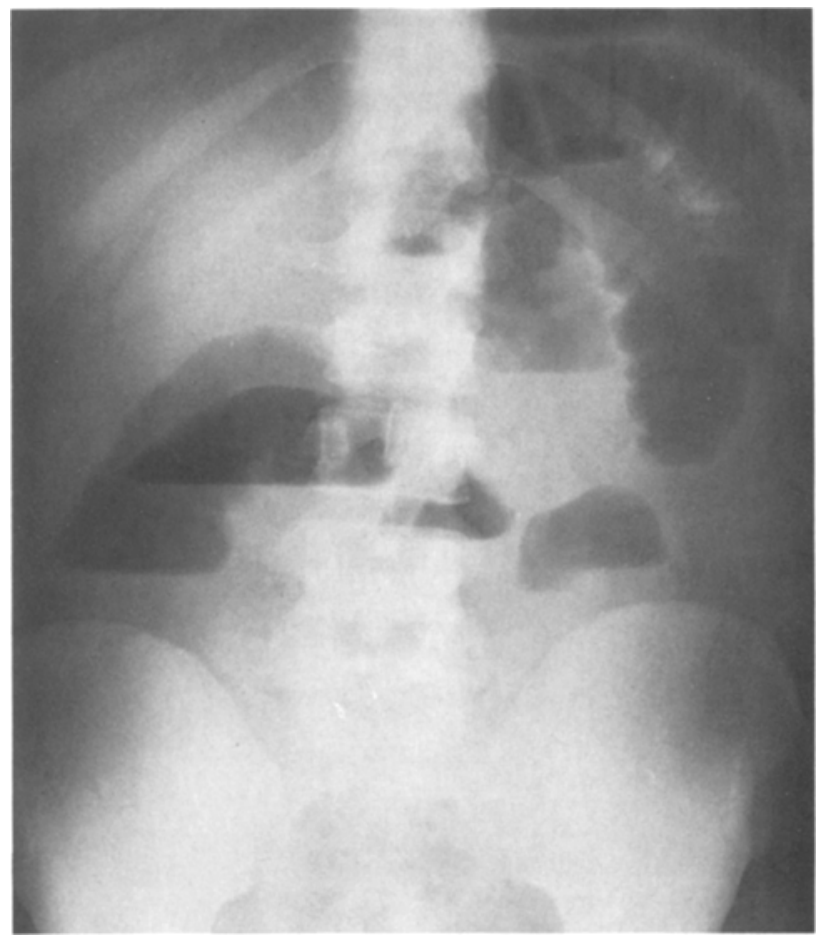

Fig. 1. An upright view of the abdomen reveals distended small bowel with multiple air-fluid levels consistent with small bowel obstruction 

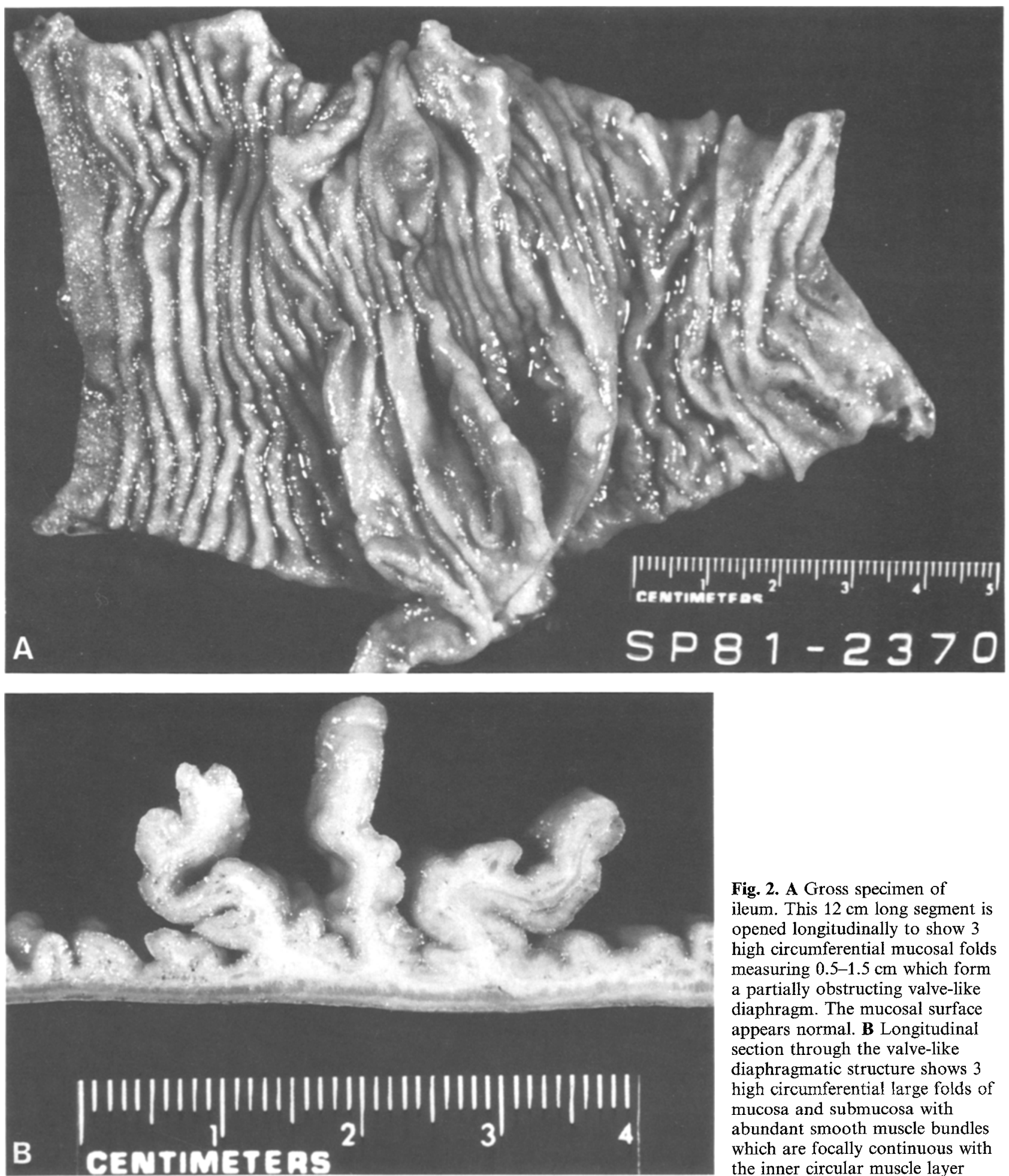

Fig. 2. A Gross specimen of ileum. This $12 \mathrm{~cm}$ long segment is opened longitudinally to show 3 high circumferential mucosal folds measuring $0.5-1.5 \mathrm{~cm}$ which form a partially obstructing valve-like diaphragm. The mucosal surface appears normal. B Longitudinal section through the valve-like diaphragmatic structure shows 3 high circumferential large folds of mucosa and submucosa with abundant smooth muscle bundles which are focally continuous with the inner circular muscle layer

\section{Discussion}

Ileal mucosal diaphragm, probably similar in etiology to antral mucosal diaphragm causing small bowel obstruction, has not been previously re- ported. Gastric antral mucosal diaphragm is often associated with partial outlet obstruction, and to date 55 cases of this entity have been reported [1-3]. However, these publications and our review of literature regarding intestinal mucosal dia- 
phragms, membranes, valve-like folds, and small bowel obstruction revealed no mention of presence of diaphragm in the ileum or colon. Duodenal diaphragm or membrane causing high intestinal obstruction is mentioned occasionally [4]. The cause of ileal diaphragm remains uncertain.

Regarding gastric mucosal diaphragm, congenital as well as acquired theories have been postulated. Rhind [5] suggested that it is an acquired anomaly produced by scarring from an annular ulceration. Congenital origin theory is based on failure of intestinal recanalization. When recanalization is incomplete, the transverse septum of mucous membrane or long plugs of epithelium may completely obliterate the intestinal lumen. Sometimes the obstructing diapharagm is perforated by several holes (fenestrated type) or by a single large central defect (iris type); these lesions give rise to incomplete obstruction [6].

In the present case 3 circumferential valvelike mucosal folds forming a diaphragm caused partial small bowel obstruction. As in most re- ported cases of gastric antral diaphragm, the symptoms of obstruction developed in early adulthood. Therefore it is postulated that ileal diaphragm is probably etiologically related to gastric mucosal diaphragm.

\section{References}

1. Felson B, Berkmen Y, Hoyumpa AM: Gastric mucosal diaphragm. Radiology 92:513-517, 1969

2. Ghahremani GG: Nonobstructive mucosal diaphragms or rings of the gastric antrum in adults. AJR 121:236-237, 1974

3. Cho KJ: Gastric antral diaphragm. Gastrointest Radiol $1: 37-40,1976$

4. Hicks MC, Kalmon EH Jr: Congenital diaphragm of the descending duodenum. Radiographic demonstration of such a lesion. Radiology 80:946-948, 1963

5. Rhind JA: Mucosal stenosis of the pylorus. $\mathrm{Br} J \mathrm{Surg}$ 46:534-540, 1959

6. Patten BM (Clark EC, ed.): Patten's Human Embryology; Elements of Clinical Development, 3rd Ed.). New York: McGraw-Hill Book Co., 1976

Received: March 23, 1982; accepted: May 25, 1982 